\title{
SpS1-High-resolution infrared spectroscopy at high and low altitudes
}

\author{
Sarah Kendrew ${ }^{1}$ \\ ${ }^{1}$ Leiden Observatory, Leiden, Netherlands \\ email: kendrew@strw.leidenuniv.nl
}

\section{Introduction}

The advantages of a high altitude, dry site for ground-based astronomy at infrared (IR) wavelengths are well-known: the lower temperature and pressure associated with increased altitude reduce the emissivities of both atmosphere and telescope, and a lower atmospheric absorption improves the transmission of IR radiation. The next generation of IR instruments under development (for ELTs) will open up a new discovery space, particularly in high-resolution (HR) spectroscopy, which will not have a space-based counterpart and has proven to be a powerful tool for studying all stages of stellar evolution (e.g. Jaffe et al., 2003). I present here a summary of quantitative work into transmission-dependent aspects of HR IR spectroscopy at high and low altitudes $\dagger$.

\section{Findings}

Modelled transmission profiles have been successfully used to improve telluric line removal in HR IR spectra (Mandell et al, 2008, Smette et al, 2009). Accurate transmission modelling is complex, requiring a detailed knowledge of vertical atmospheric profiles in $\mathrm{T}, \mathrm{P}$ and molecular constituents. The combination of models and experimental data can lead to a better understanding of the interplay between these factors.

The largest transmission gains with altitude are found in spectral regions dominated by water absorption; species with high abundances in the upper atmosphere, such as $\mathrm{O}_{3}$, show only a moderate improvement. The regions benefiting the most from high and dry sites are the 6.5 $\mu \mathrm{m}$ region of M-band, between 6.5-8 $\mu \mathrm{m}$ (N-band) and the entire Q-band (17-25 $\mu \mathrm{m})$, which is dominated by water line absorption.

The relative velocity between Earth and a (galactic) science target can be used to Dopplershift diagnostic spectral lines from behind their telluric equivalent. This technique is commonly used for e.g. $13.7 \mu \mathrm{m} \mathrm{C}_{2} \mathrm{H}_{2}$ absorption (also for $4.7 \mu \mathrm{m} \mathrm{CO}$ emission), where an improvement of $25 \%$ in transmission can result from this method (depending on the target co-ordinates). In targets that push the limits of the instrumental sensitivity the observing efficiency is much improved when observing from a high-altitude site.

\section{References}

Jaffe et al., 2003, ApJ 596(2), 1053

Mandell, A. et al., 2008, ApJ 681, L25

Smette, A. et al., 2009, these proceedings

$\dagger$ Work presented here uses the 2008 edition of HITRAN, the Reference Forward Model (RFM), more information: http://www.atm.ox.ac.uk/RFM/index.html, and a standard tropical atmosphere profile. 